\title{
Associations Between Insulin Resistance and Adverse Pregnancy Outcomes in Women With Gestational Diabetes Mellitus: a Retrospective Study
}

\section{Jing Lin}

The Second School of clinical Medicine, Southern Medical University, Guangzhou

Hua Jin

Naval Clinical College, Anhui Medical University, Anhui

Lei Chen ( 1329695616@qq.com)

The Second School of clinical Medicine, Southern Medical University, Guangzhou

\section{Research Article}

Keywords: gestational diabetes mellitus, insulin resistance, pregnancy outcomes

Posted Date: February 24th, 2021

DOl: https://doi.org/10.21203/rs.3.rs-235578/v1

License: (c) (i) This work is licensed under a Creative Commons Attribution 4.0 International License.

Read Full License

Version of Record: A version of this preprint was published at BMC Pregnancy and Childbirth on July 23rd, 2021. See the published version at https://doi.org/10.1186/s12884-021-04006-x. 


\section{Abstract}

Background: This study aimed to explore the relationship between insulin resistance (IR) and adverse pregnancy outcomes in women with gestational diabetes mellitus (GDM), and to determine the risk factors for IR in women with GDM.

Methods: This study employed a retrospective survey of 710 women diagnosed with GDM. Serum lipids, fasting plasma glucose (FPG), glycosylated hemoglobin (HbA1c), and serum protein were measured in the first trimester (6-12 weeks), and OGTT and fasting insulin tests were performed in the second trimester (24-28 weeks). These results were then used to evaluate IR by homeostasis model assessment (HOMA). When HOMA-IR $\geq 2.0$, IR was diagnosed. The relationship between HOMA-IR and adverse pregnancy outcomes was analyzed by a logistic regression model, and multiple stepwise regression was used to analyze the risk factors of HOMA-IR.

Results: GDM with IR was significantly associated with the hypertensive disorders of pregnancy and large for gestational age (LGA) ( $P=0.002,0.012$, respectively). Body mass index (BMI) before pregnancy, FPG, $\mathrm{HbA} 1 \mathrm{c}$, and total triglyceride $(\mathrm{TG})$ in early pregnancy were all significantly positively correlated with IR in the second trimester $(P<0.05)$, while age was significantly negatively correlated with $\mathrm{IR}(P<0.001)$.

Conclusion: GDM combined with IR in the second trimester increases adverse pregnancy outcomes, especially the risk of hypertensive disorders of pregnancy and LGA. In addition, BMI before pregnancy, FPG, HbA1c, and TG in early pregnancy are all independent risk factors for IR.

\section{Highlights}

- Increasing IR in the second trimester predicts an increased risk of hypertensive disorders of pregnancy and LGA in women with GDM.

- BMI before pregnancy, FPG, HbA1c, and TG in early pregnancy are all independent risk factors for the development of IR in the second trimester.

\section{Introduction}

Gestational diabetes (GDM) is a common disease during pregnancy. According to a report from the International Diabetes Federation (IDF) in 2017, $14 \%$ of pregnant women suffer from it worldwide (1). In China, the incidence of GDM has reached $17.5 \%$, with an increasing growth trend (2). GDM can bring about some short-term and long-term disorders to women and their babies, which has attracted increasing attention from the entire society. In practice, once GDM is diagnosed, individualized treatment plans involving diet control, appropriate regular exercise, and insulin intervention are routinely made immediately by doctors for patients. However, it has been found that even when the recommended plans are well followed, the pregnancy outcomes cannot be effectively improved (3). Some researches ascribes this to insulin resistance $\mathrm{IR} \rrbracket(4)$ and the heterogeneity of IR in pregnant women with GDM somehow results in different outcomes $(5,6)$. Therefore, more exploration on the relationship between IR in the 
second trimester (24-28 weeks) and adverse pregnancy outcomes in GDM women may help identify women who are at high risk of these adverse outcomes, and management can therefore be carried out in early pregnancy even pre-pregnancy, sometimes adding insulin sensitization therapy if necessary to improve the prognosis.

This study aimed to determine whether IR in the second trimester is associated with certain specific adverse pregnancy outcomes, and to further navigate the risk factors that can predict IR in in the second trimester in Chinese GDM women.

\section{Methods}

\section{Material and Methods}

A retrospective analysis was made. It was based on $710 \mathrm{GDM}$ women who were treated at the Obstetrics Clinic of the Sixth Medical Center of PLA General Hospital from January 1, 2018 to December 31, 2019. The inclusion criteria were as follows: GDM was diagnosed by a $75 \mathrm{~g}$ oral glucose tolerance test (OGTT) between 24-28 weeks of pregnancy. GDM was diagnosed using the standards of the International Association of Diabetes and Pregnancy Study Group (IADPSG) (7). Women with pre-gestational diabetes, diabetes diagnosed before 24 weeks of pregnancy, multiple pregnancies, chronic hypertension, and women who gave birth before 28 weeks were excluded from the study. After diagnosis, all the GDM women were referred to the nutrition clinic to receive guidance on GDM diet and exercise. After 7 days of self-adjustment to their diet and activities, venous blood was drawn to check the blood sugar before and 2 hours after meals, and based on the results the nurse would further adjust their diet and physical activity plans and teach them blood glucose monitoring at home. Generally, insulin is recommended for regulation when fasting plasma glucose is higher than $5.3 \mathrm{mmol} / \mathrm{L}$, and 2 hours postprandial blood glucose is higher than $6.7 \mathrm{mmol} / \mathrm{L}$.

The basic information of the pregnant women was collected by an obstetrician, including the woman's age, weight before pregnancy, weight at delivery, height, gestational week of delivery, delivery method, outcome, newborn weight, and other relevant parameters. The weight before pregnancy was measured during the pre-pregnancy check-up. If there was no pre-pregnancy check-up data, it was taken at the first pregnancy check-up. The weight at delivery was collected when they were admitted to the hospital for delivery. The total weight gain during pregnancy (GWG) was calculated by subtracting the weight before pregnancy from the weight at delivery. The newborns' basic information was recorded immediately after birth. All the data and information related to pregnancy outcomes was extracted from electronic medical records. What we were primarily concerned with was the following conditions: cesarean section, premature delivery, premature rupture of membranes, hypertensive disorders of pregnancy, postpartum hemorrhage, macrosomia, small for gestational age (SGA), and large for gestational age (LGA).

\section{Several diagnostic criteria}


Preterm delivery: delivery between 28 and 37 weeks of gestation. Premature rupture of membranes $\rrbracket a$ spontaneous rupture of membranes that occurs before delivery. Hypertensive disorders of pregnancy hypertension during pregnancy, preeclampsia, and eclampsia. Postpartum hemorrhage $₫ F o r$ vaginal delivery $\bigotimes$ the bleeding volume exceeded $500 \mathrm{~mL}$ within 24 hours after delivery of the fetus; for cesarean section the bleeding volume exceeded $1,000 \mathrm{~mL}$. Small for gestational age infant[SGADand large for gestational age infant[LGADthe newborns whose birth weights were below the $10^{\text {th }}$ percentile and above the $90^{\text {th }}$ percentile of the average weight of the same gestational age, with the 2015 Chinese neonatal birth weight curve for different gestational ages used as reference (8). Macrosomia: the birth weight of the newborn $\geq 4,000 \mathrm{~g}$.

\section{Laboratory methods}

Peripheral venous blood was drawn during the first trimester (6-12 weeks of pregnancy) and the second trimester (24-28 weeks of pregnancy), after the GDM women had fasted for 8-12 hours over night. The blood sample was centrifuged at 3,000 rpm for $10 \mathrm{~min}$ to obtain serum for analysis. In the first trimester, all samples were tested for serum FPG, HbA1c, total cholesterol (TC), TG, total protein (TP), and albumin levels (ALB). All samples from 24-28 weeks of gestation were tested for serum fasting insulin, fasting blood glucose, and blood sugar level both 1 hour and 2 hours after orally taking glucose. Fasting insulin was tested via electrochemiluminescence immunoassay (ROCHE COBASE 601). Serum blood glucose was tested by the hexokinase method, triglyceride level was tested by the peroxidase method, cholesterol was tested by the coupling-POD enzyme colorimetric method, serum total protein by the biuret method, and albumin by bromomethylolate green. All the tests were performed in an automatic biochemical analyzer (ROCHE COBASE 8000).

The homeostasis model assessment (HOMA) was used to evaluate IR and $\beta$-cell function in GDM. HOMA$\mathrm{IR}=$ fasting plasma insulin concentration $(\mu \mathrm{U} / \mathrm{mL}) \times$ fasting plasma glucose $(\mathrm{mmol} / \mathrm{L}) / 22.5 . \mathrm{HOMA}-\beta=$ $20 \times$ fasting plasma insulin concentration $(\mu \mathrm{U} / \mathrm{mL}) /$ (fasting plasma glucose [mmol/L]-3.5). According to the HOMA value, the cut-off value was set at 2.0 , and then the patients were divided into the non-IR GDM group (HOMA<2) and IR GDM group (HOMA-IR $\geq 2)(9)$.

\section{Statistical analysis}

SPSS 23.0 was used for statistical analysis. The Shapiro-Wilk test was used to determine the data normality. For continuous variable data in a normal distribution, the mean and standard deviation were chosen for description, and the t-test for comparison between groups. For the continuous variable data in a skewed distribution, the median and quartile were chosen, and the Mann-Whitney $\mathrm{U}$ test was used for comparison between groups. Univariate and multivariate logistic regression models were used to analyze the relationship between HOMA-IR and adverse pregnancy outcomes, and the multiple regression model was used to analyze the relationship between risk factors and HOMA-IR. All tests were statistically significant when $\mathrm{p}<0.05$. 


\section{Results}

\section{General characteristics of the mothers and newborns among the compared groups}

710 GDM women were included, aging from 23 to 51 years old with an average age of $33.00 \pm 4.18$ years old. All of them made regular medical visits to the Obstetrics Clinic of the Sixth Medical Center of PLA General Hospital. Their gestational week of delivery ranged from 32 to 42 weeks, with an average of $38.13 \pm 1.49$ weeks.

Table 1 shows the clinical characteristics of GDM women and their newborns $\ a n d$ there was no difference in the age distribution between the two groups. The pre-pregnancy BMI $(P<0.001)$ was statistically different, significantly higher in the IR GDM group. For metabolic parameters, the FPG $(P<0.001), \mathrm{HbA} 1 \mathrm{c}(P<0.001), \mathrm{TC}(P=0.003)$, and TG $(P<0.001)$ of IR GDM women during early pregnancy were significantly higher than those of the non-IR GDM group. However, TP and ALB were similar between the two groups $(P=0.061, P=0.835)$. Pancreatic $\beta$-cell function in the IR GDM group was significantly enhanced $(P<0.001)$. Only 12 women received intensive insulin therapy based on diet control and diabetic education, and all of them were from the IR GDM group. With 30 premature birth cases excluded, there was a statistically significant difference in the birth weight of the newborns between the two groups $(P=0.014)$, but there was no significant difference in GWG $(P=0.638)$.

\section{Risk factors of IR}

Meanwhile, the risk factors for IR were explored. Multiple stepwise regression analyses were performed with independent variables including age, pre-pregnancy BMI, FPG, HbA1c, TP, ALB, TC, and TG. It can be seen from the data in Table 2, the statistically significant factors were age, pre-pregnancy BMI, FPG, and TG in early pregnancy $(P<0.05)$.

\section{The relationship between HOMA-IR and adverse pregnancy outcomes}

It can be seen from the data in Table 3 that IR did not significantly increase the risk of GDM cesarean section, premature delivery, premature rupture of membranes, postpartum hemorrhage, macrosomia, and SGA $(P>0.05)$. After adjusting for confounding factors, the ORs $(95 \% \mathrm{Cl})$ of hypertensive disorders of pregnancy and LGA were $5.883(1.885,18.366)$ and $1.655(1.112,2.463)$, respectively.

\section{Disscussion}

\section{1) IR harms the prognosis of GDM women}

From the results of this retrospective study, IR was found to be harmful to the GDM's prognosis, with an increase in adverse outcomes. When the confounding factors were excluded, the incidences of hypertensive disorders of pregnancy and LGA were significantly higher.

i. GDM with IR and hypertensive disorders of pregnancy 
In our study, compared with the GDM women without IR, the risk of hypertensive disorders of pregnancy increased up to 5 times in GDM women with IR. An Iranian study (10) had similar findings: insulin resistance, closely related to preeclampsia, served as a risk factor in the process. Indeed, before clinical symptoms occur, the fasting insulin level increases accordingly as the disease progresses. Based on this, identifying GDM women with IR could be considered as a starting point for the clinical prevention of hypertensive disorders of pregnancy. However, the specific mechanism is still unclear. In the mainstream opinion, physiological IR provides more glucose to the fetus to better nourish its development, but overactivation of IR in pregnancy leads to decreased carbon monoxide (NO), discorded lipid metabolism, low prostaglandin E2 synthesis, and damaged vascular endothelial cells, eventually resulting in maternal high blood pressure $(11,12)$.

\section{ii. GDM with IR and LGA}

GDM with IR may also cause fetal overgrowth. In our study, babies born to GDM women with IR had a significant increase in average birth weight compared with the babies of GDM women without IR. With the confounding factors (age, BMI, TG, FPG, HbA1c, etc.) adjusted, IR was found to be an independent risk factor of LGA, doubling the risk. This discovery is similar to that of Yamashita H's research(13), in which HOMA-IR was positively correlated to the birth weights of newborns. The mechanism of this phenomenon has been partially explained. In Pedersen's (14) hypothesis, maternal hyperglycemia passing through the placental barrier stimulates the fetus to secrete more insulin which promotes fat and protein accumulation, leading to excessive fetal growth. As a result, high-level maternal insulin, which though cannot go through the placenta, causes changes in the placenta's metabolism and gene expression, eventually disturbing fetal growth.

\section{2) IR's risk factors}

Previous research has mainly focused on the risk factors of IR or GDM, but few referred to the case of GDM with IR, which we analyzed in our study. Based on our data, BMI before pregnancy, TG, HbA1c, and FPG in early pregnancy were considered to be the risk factors of GDM with IR. Our results are similar to the results of Sun's study (5), in which BMI and TG were related to GDM with IR in the second trimester, but FPG and $\mathrm{HbA} 1 \mathrm{c}$ were not. The differences in the results possibly derive from the fact that our FPG and $\mathrm{HbA} 1 \mathrm{c}$ were tested in early pregnancy, while Sun's results were obtained from the second trimester. Meanwhile, it is worth noting that FPG and $\mathrm{HbA} 1 \mathrm{c}$, although within normal range, were higher in GDM women with IR than that in those without IR, which indicates that more benefit will occur if blood sugar control is performed early. However, considering the dangers of hypoglycemia, more research needs to be done to determine the appropriate control range.

Furthermore, different from Rodríguez-Morán M's study (15), in our research, age was a protective factor for GDM with IR. We consider that this difference in results comes from the fact that we focused on GDM women, whereas his conclusion was based on an investigation of non-pregnant IR women. Therefore, related population-based studies on larger scales are needed to confirm the results. 


\section{3) Management of GDM with IR}

In this study, it was found that the HOMA- $\beta$ value is increased in GDM women with IR, which is considered to result from low efficiency of insulin utilization in tissue and the enhanced compensatory secretion function of islet $\beta$-cells. The protection of islet $\beta$-cell function is closely related to the reduction of endogenous insulin demand (16). Therefore, women with insulin resistance to GDM are likely to benefit from insulin sensitization therapy, providing a new approach for the individualized treatment of GDM. This view is similar to that of a study in Poland, in which Sokup (17) suggested that GDM women with HOMA $>1.29$ should be treated with improved tissue sensitivity to insulin, while GDM women with HOMA>2.89 should be treated with metformin combined with insulin.

It is worth noting that the current clinical management goal of GDM is blood glucose. In fact, a high blood glucose level can only explain a small part of the variation in birth weight (18). In this study, although the most of women with GDM had a normal blood glucose range, women with GDM with insulin resistance had a higher risk of LGA. Therefore, insulin resistance should also be an important indicator in pregnancy monitoring, especially for GDM women within the normal range of blood glucose and with high risk factors.

In particular, in this study, only $1.7 \%$ of the GDM women needed to control blood glucose by insulin treatment, which is quite different from in other countries. In Australia, this proportion can reach to $52.8 \%$ (19). This difference is considered to result from the lack of consensus and consistency in the screening and diagnostic criteria for gestational diabetes in various countries. In China, the diagnosis of GDM is up to the more rigid IADPSG standard, in which the cut-off value is lower than in other guidelines. GDM can be diagnosed by only an abnormal blood glucose reading. However, alternative standards are still used in some centers and countries including Australia (20). This has led to the expansion of the GDM population in China, while the proportion of women who really need insulin treatment has decreased. Therefore, it is worth exploring whether the IADPSG standard is applicable to the Asian population. We further suggest an appropriate stratification of the management of GDM women based on HOMA-IR to reduce the loss of medical resources.

\section{4) Limitations}

First, this study was a retrospective study, in which bias was inevitably introduced in the participant selection. Second, our research subjects were GDM pregnant women, so the results would not be applicable to non-GDM women. Third, our model did not include other risk factors that affect IR, including diet, physical activity, family history, and past history. In addition, HOMA-IR before childbirth was not tested, so it was not clear whether the women's IR was improved by lifestyle and diet management. In the end, more research is needed to dynamically observe the changes in IR before pregnancy, during pregnancy, and after delivery, and to clarify the influencing factors of IR and the relationship between IR and GDM. 


\section{Conclusion}

In summary, our research shows that IR in the second trimester is associated with adverse pregnancy outcomes like hypertensive disorders of pregnancy and LGA. Moreover, pre-pregnancy BMI, TG, FPG, and $\mathrm{HbA} 1 \mathrm{c}$ in the first trimester are independent risk factors for IR in the second trimester. For women of childbearing age with one of these factors, it is strongly recommended that they actively lose weight and avoid a high-sucrose, high-fat diet before pregnancy. In addition, for GDM women with IR, stricter weight management and insulin sensitivity treatment in the mid-and late-gestational periods are suggested to further avoid adverse outcomes.

\section{Abbreviations}

IR: Insulin resistance; GDM: Gestational diabetes mellitus; FPG: Fasting plasma glucose; HbA1c: Glycosylated hemoglobin; TC: Serum total cholesterol; TG: Serum total triglyceride; TP: Serum total protein; ALB: Serum albumin; BMI: Body mass index; SGA: Small for gestational age infant; LGA: Large for gestational age infant; HOMA-IR: Homoeostasis model assessment of IR; HOMA- $\beta$ :Homoeostasis model assessment of $\beta$-cell function; GWG: Total weight gain during pregnancy; OGTT: $75 \mathrm{~g}$ oral glucose tolerance test.

\section{Declarations}

\section{Ethics approval and consent to participate}

Institutional Review Board Project \# No. HZKY-PJ-2020-42 was approved on 12 May 2020 by the Sixth Medical Center of PLA General Hospital Ethics Committee. All study participants gave verbal informed consent, this was approved by the Sixth Medical Center of PLA General Hospital Ethics Committee as it was a retrospective study and no interventions was given to the participants.This manuscript reported adherence to Declaration of Helsinki.

\section{Consent for publication}

Not applicable.

\section{Availability of data and materials}

The dataset used in the present study is available from the corresponding author upon reasonable request.

\section{Competing interests}

The authors declare they have no competing interests.

\section{Funding}


This work was supported by Capital's Funds for Health Improvement and Research, (CFH) in People's Republic of China (020-1-5112).

\section{Author Contributions}

LJ: Data curation, Methodology, Writing - original draft. JH: collected data and contributed to data cleaning and analysis. CL: Conceptualization, Funding acquisition, Project administration, Writing - review \& editing.

\section{Acknowledgements}

We appreciate all the investigators'efforts in data collection.

\section{Financial Disclosure}

The authors have no financial relationships relevant to this article to disclose.

\section{References}

1. Q\&A: Key points for IDF Diabetes Atlas 2017. Diabetes Res Clin Pract. 2018; 135: 235-36.

2. Zhu WW, Yang HX, Wei YM, et al. Evaluation of the value of fasting plasma glucose in the first prenatal visit to diagnose gestational diabetes mellitus in china. Diabetes Care. 2013; 36(3): 586-90.

3. Griffith RJ, Alsweiler J, Moore AE, et al. Interventions to prevent women from developing gestational diabetes mellitus: an overview of Cochrane Reviews. Cochrane Database Syst Rev. 2020; 6(6): Cd012394.

4. Ernst $S$, Demirci $C$, Valle $S$, et al. Mechanisms in the adaptation of maternal $\beta$-cells during pregnancy. Diabetes Manag (Lond). 2011; 1(2): 239-48.

5. Sun $Y Y$, Juan J, Xu QQ, et al. Increasing insulin resistance predicts adverse pregnancy outcomes in women with gestational diabetes mellitus. J Diabetes. 2020; 12(6): 438-46.

6. Naseh A, Nourbakhsh S, Tohidi M, et al. Associations between anthropometric characteristics and insulin markers in mothers and their neonates and with neonate`s birth weight: An observational cohort study. Turk J Pediatr. 2017; 59(6): 625-35.

7. Chiefari E, Arcidiacono B, Foti D, et al. Gestational diabetes mellitus: an updated overview. J Endocrinol Invest. 2017; 40(9): 899-909.

8. Zhu L, Zhang R, Zhang S, et al. [Chinese neonatal birth weight curve for different gestational age]. Zhonghua Er Ke Za Zhi. 2015; 53(2): 97-103.

9. Lee CH, Shih AZ, Woo YC, et al. Optimal Cut-Offs of Homeostasis Model Assessment of Insulin Resistance (HOMA-IR) to Identify Dysglycemia and Type 2 Diabetes Mellitus: A 15-Year Prospective Study in Chinese. PLoS One. 2016; 11(9): e0163424.

10. Abhari FR, Ghanbari Andarieh M, Farokhfar A, et al. Estimating rate of insulin resistance in patients with preeclampsia using HOMA-IR index and comparison with nonpreeclampsia pregnant women. 
Biomed Res Int. 2014; 2014: 140851.

11. Villalobos-Labra R, Silva L, Subiabre M, et al. Akt/mTOR Role in Human Foetoplacental Vascular Insulin Resistance in Diseases of Pregnancy. J Diabetes Res. 2017; 2017: 5947859.

12. Harskamp RE and Zeeman GG. Preeclampsia: at risk for remote cardiovascular disease. Am J Med Sci. 2007; 334(4): 291-5.

13. Yamashita $\mathrm{H}$, Yasuhi I, Fukuda $\mathrm{M}$, et al. The association between maternal insulin resistance in midpregnancy and neonatal birthweight in uncomplicated pregnancies. Endocr J. 2014; 61(10): 1019-24.

14. Kc K, Shakya S and Zhang H. Gestational diabetes mellitus and macrosomia: a literature review. Ann Nutr Metab. 2015; 66 Suppl 2: 14-20.

15. Rodríguez-Morán M and Guerrero-Romero F. Insulin resistance is independently related to age in Mexican women. J Endocrinol Invest. 2003; 26(1): 42-8.

16. Xiang AH, Peters RK, Kjos SL, et al. Effect of pioglitazone on pancreatic beta-cell function and diabetes risk in Hispanic women with prior gestational diabetes. Diabetes. 2006; 55(2): 517-22.

17. Sokup A, Ruszkowska-Ciastek B, Góralczyk K, et al. Insulin resistance as estimated by the homeostatic method at diagnosis of gestational diabetes: estimation of disease severity and therapeutic needs in a population-based study. BMC Endocr Disord. 2013; 13: 21.

18. Yang $X$, Zhang $H$, Dong $L$, et al. The effect of glucose levels on fetal birth weight: a study of Chinese gravidas in Tianjin, China. J Diabetes Complications. 2004; 18(1): 37-41.

19. Wong VW and Jalaludin B. Gestational diabetes mellitus: who requires insulin therapy? Aust N Z J Obstet Gynaecol. 2011; 51(5): 432-6.

20. Plows JF, Stanley JL, Baker PN, et al. The Pathophysiology of Gestational Diabetes Mellitus. Int J Mol Sci. 2018; 19(11).

\section{Tables}


Table 1

General characteristics comparison

\begin{tabular}{|lllll|}
\hline & Non-IR GDM & IR GDM & $t$ & $P$ \\
\hline Number & 418 & 292 & & \\
\hline Age & $33.20 \pm 4.127$ & $32.72 \pm 4.255$ & 1.495 & 0.135 \\
\hline Pre-pregnant BMI(kg/m2) & $21.42 \pm 2.59$ & $24.15 \pm 3.68$ & -10.937 & $<0.001$ \\
\hline FPB (mmol/L) & $4.70 \pm 0.44$ & $4.85 \pm 0.49$ & -4.145 & $<0.001$ \\
\hline HbA1c (\%) & $5.26 \pm 0.28$ & $5.38 \pm 0.30$ & -5.684 & $<0.001$ \\
\hline HOMA- $\beta$ & $85.64 \llbracket 68.94,110.67 \rrbracket$ & $141.36 \rrbracket 114.13,182.18 \rrbracket$ & & $<0.001$ \\
\hline Serum Lipid & & & -2.985 & 0.003 \\
\hline TC(mmol/L) & $4.35 \pm 0.83$ & $4.55 \pm 0.93$ & -3.792 & $<0.001$ \\
\hline TG(mmol/L) & $1.23 \pm 0.92$ & $1.59 \pm 1.46$ & & \\
\hline Serum protein & & & -1.873 & 0.061 \\
\hline TP(mmol/L) & $73.0 \pm 4.13$ & $73.57 \pm 3.90$ & 0.209 & 0.835 \\
\hline ALB(mmol/L) & $42.53 \pm 3.15$ & $42.48 \pm 2.83$ & -0.47 & 0.638 \\
\hline aGWG (kg) & $10.77 \pm 4.53$ & $10.96 \pm 5.34$ & -2.461 & 0.014 \\
\hline aBirth weight (g) & $3343.60 \pm 390.24$ & $3423.77 \pm 434.55$ & & \\
\hline
\end{tabular}

a Non-IR GDM group (n 404), IR GDM group (n 276) 『with 30 cases of preterm birth excluded

Table 2

Multiple stepwise regression analysis between HOMA-IR and various indicators

\begin{tabular}{|lllllll|}
\hline Variable & $\beta$ & $S E$ & $\beta^{\prime}$ & $t$ & $P$ & $95 \% \mathrm{Cl}$ \\
\hline Constant & -3.734 & 0.823 & & -4.535 & $<0.001$ & $-5.351 \sim-2.118$ \\
\hline Age & -0.035 & 0.010 & -0.121 & -3.704 & $<0.001$ & $-0.017 \sim-0.051$ \\
\hline Pre-pregnant BMI & 0.149 & 0.012 & 0.405 & 12.02 & $<0.001$ & $0.124 \sim 0.173$ \\
\hline TG & 0.195 & 0.034 & 0.189 & 5.745 & $<0.001$ & $0.128 \sim 0.261$ \\
\hline FPB & 0.286 & 0.087 & 0.108 & 3.288 & 0.001 & $0.115 \sim 0.457$ \\
\hline HbA1c & 0.383 & 0.138 & 0.92 & 2.783 & 0.006 & $0.113 \sim 0.654$ \\
\hline
\end{tabular}


Table 3

Influence of HOMA-IR on pregnancy outcome of GDM pregnant women

\begin{tabular}{|c|c|c|c|c|}
\hline & \multicolumn{2}{|c|}{ Univariate logistic regression } & \multicolumn{2}{|l|}{$\begin{array}{l}\text { Multivariate logistic } \\
\text { regression }\end{array}$} \\
\hline & OR\} 9 5 \% \mathrm { Cl } / $&{P} &{\text { ORD95\% CII }} &{P} \\
{\hline \text { Cesarean section }} &{1.262(0.924,1.724)} &{0.143} &{1.170(0.805,1.699)} &{0.411} \\
{\hline \text { Premature delivery }} &{1.802(0.853,3.805)} &{0.123} &{\text { 1.992(0.828,4.791) }} &{0.124} \\
{\hline \text { Premature rupture of membranes }} &{1.316(0.931,1.862)} &{0.120} &{1.607(0.716,1.590)} &{0.750} \\
{\hline \text { Postpartum hemorrhage }} &{1.122(0.724,1.739)} &{0.607} &{\text { 1.207(0.731,1.992) }} &{0.462} \\
{\hline \begin{array}{l}\text { Hypertensive disorders of } \\
\text { pregnancy }\end{array}} &{\text { 8.398(3.193,22.072 }} &{<0.001} &{5.883(1.885,18.366)} &{0.002} \\
{\hline \text { Macrosomia }} &{1.621(0.868,3.029)} &{0.130} &{1.073(0.517,2.226)} &{0.850} \\
{\hline \text { LGA }} &{2.037(1.436,2.889)} &{<0.001} &{1.655(1.112,2.463)} &{0.013} \\
{\hline \text { SGA }} &{1.563(0.742,3.289)} &{0.24} &{1.365(0.561,3.323)} &{0.493} \\
$\hline
\end{tabular}

Confounding factors: age, pre-pregnancy BMI, FPG, HbA1c, TC, TG. 